\title{
Landau Damping of Surface Plasma Waves
}

\author{
Myoung-Jae Lee and Hee J. Lee*
}

Department of Physics, Hanyang University, Seoul 133-791, Korea

\begin{abstract}
The integral of the kinetic electrostatic surface wave dispersion relation is asymptotically evaluated to determine the frequencies of the normal modes and the corresponding Landau damping for the high frequency surface electron plasma wave and the low frequency surface ion acoustic wave. The asymptotically calculated Landau damping rates apply to the limiting cases of $k_{x} \lambda_{e}<<1$ and $k_{x} \lambda_{e} \gg>1$ where $k_{x}$ is the wavenumber and $\lambda_{e}$ is the electron Debye length. We also calculate Landau damping rate of the transverse magnetic (TM) mode surface wave.
\end{abstract}

Keywords: Surface plasma wave, Landau damping.

\section{INTRODUCTION}

In contrast to Landau damping accompanying bulk plasma waves which can be immediately evaluated in terms of the well-documented plasma dispersion function, Landau damping in surface plasma waves is obtained only after carrying out the integral of the surface wave dispersion relation which requires basically numerical computation. Analytic results can be obtained only after resorting to some approximation to deal with the integral which involves complex algebraic structure and Maxwellian distribution function contained in the plasma dielectric function $\left(\varepsilon_{L}\right)$. This is seen from the dispersion relation integral

$1+\frac{k_{x}}{\pi} \lim _{z \rightarrow 0} \int d k_{z} \frac{e^{i k_{z} z}}{k^{2} \varepsilon_{L}}=1+\frac{k_{x}}{\pi} \int_{-\infty}^{\infty} \frac{d k_{z}}{k^{2} \varepsilon_{L}}=0$.

Equation (1) is the well-known electrostatic surface wave dispersion relation integral [1-3] which applies to a semiinfinite Vlasov-Poisson plasma separated from a vacuum by the interface $z=0$ under the specular reflection boundary condition. In Eq. (1), we assumed that the Fourier variable, the wave vector, $k=\left(k_{x}, 0, k_{z}\right)$, which replaces the spatial coordinate in Vlasov and Maxwell equations. Putting $k_{y}=0$ is not loss of generality. The geometry which is involved in surface wave has two directions: the perpendicular direction to the interface (the $z$-coordinate here) and the direction of the propagation of the surface wave which is taken to be the $x$-direction here. We wrote the first expression of Eq. (1) to show that the pole at the denominator is associated with the attenuation constant of the surface wave. Otherwise, the second equality is the object of our analysis.

In this work, it is shown that there are transformations which facilitate asymptotic evaluation of the integral with sufficient rigor in the respective parameter regions of

*Address correspondence to this author at the 107-2201 Buyoung Apt., Donong-dong Namyangju-City, Kyunggi-do 472-709, Korea; Tel: +82-22220-1983; Fax: +82-2-2299-2028; E-mail: ychjlee@yahoo.com $k_{x} \lambda_{e}<<1$ and $k_{x} \lambda_{e}>1$. With the transformations, we expect to obtain almost exact results in the aforementioned parameter regions, without expending labor of numerical work.

Here we consider two normal modes of surface wave: high frequency electron plasma surface wave and low frequency ion acoustic surface wave. The high frequency surface wave (as well as its corresponding bulk wave) is such that $\omega \gg k \cdot v_{e}$ while the low frequency ion acoustic surface wave as well as its corresponding bulk ion acoustic wave has frequencies such that $k \cdot v_{i}<<\omega<<k \cdot v_{e} \quad\left(v_{\alpha}\right.$ is the thermal velocity of species $\alpha$ ). Using the expressions for $\varepsilon_{L}$ in the respective frequency ranges, given in standard texts, we integrate Eq. (1) to determine real frequency and the Landau damping in the following sections.

Landau damping rates of the surface waves are reported in the monographs written by Alexandrov et al. [1] and Sitenko [4]; with very unsimilar results. The longitudinal dielectric function $\varepsilon_{L}$ contains electron term as well as ion term (see Eq. (3)). Alexandrov et al. [1] neglected the ion term while keeping the electron term. Sitenko [4] opted for the opposite choice. In this work, both terms are included, and the relative importance of the two terms is weighed. Correctness of this work is checked against these earlier results.

We also calculate in the last section Landau damping rate of TM mode surface wave (surface polariton) in two asymptotic regions $\left(\omega_{p e} / k<<c\right.$ and $\left.\omega_{p e} / k>>c\right)$.

\section{SURFACE ION ACOUSTIC WAVE}

Let us first consider the surface ion acoustic wave. In the low frequency range, we have $\varepsilon_{L}=\varepsilon_{r}+i \varepsilon_{i}$ with $[1,5]$

$$
\varepsilon_{r}=1+\frac{1}{k^{2} \lambda_{e}^{2}}\left(1-\frac{T_{e}}{m_{i}} \frac{k^{2}}{\omega^{2}}\right)=1-\frac{\omega_{p i}^{2}}{\omega^{2}}+\frac{1}{k^{2} \lambda_{e}^{2}}
$$


$\varepsilon_{i}=\sum_{\alpha=e, i} \frac{\sqrt{\pi}}{k^{2} \lambda_{\alpha}^{2}} \frac{\omega}{k} \sqrt{\frac{m_{\alpha}}{2 T_{\alpha}}} \exp \left[\frac{-m_{\alpha} \omega^{2}}{2 T_{\alpha} k^{2}}\right]$

Our general procedure toward obtaining the surface wave dispersion relation and the Landau damping is as follows. Assuming $\varepsilon_{r} \gg \varepsilon_{i}$, we adopt a kind of perturbation approach: the zero order dispersion relation would be obtained by neglecting $\varepsilon_{i}$ in Eq. (1). The real frequency determined only by $\varepsilon_{r}$ would be used, if necessary, to simplify the integral. We put $\varepsilon_{L}=\varepsilon_{r}\left(1+i \varepsilon_{i} / \varepsilon_{r}\right)$ where the second term is $<<1$. Then the dispersion relation integral in Eq. (1) becomes

$1+\frac{k_{x}}{\pi} \int \frac{d k_{z}}{k^{2} \varepsilon_{r}}\left(1-i \frac{\varepsilon_{i}}{\varepsilon_{r}}\right)=0$. part,

Approximate eigenfrequency is obtained from the real

$D_{r} \equiv 1+\frac{k_{x}}{\pi} \int \frac{d k_{z}}{k^{2} \varepsilon_{r}}=0$

which takes the form upon using Eq. (2)

$1+\frac{k_{x}}{\pi q(\omega)} \int \frac{d k_{z}}{k_{z}^{2}+\gamma^{2}}=0$

$q(\omega)=1-\frac{\omega_{p i}^{2}}{\omega^{2}}, \gamma=\sqrt{k_{x}^{2}+\frac{1}{q \lambda_{e}^{2}}}$

where $\omega_{p i}=\sqrt{\frac{4 \pi N e^{2}}{m_{i}}}$ is ion plasma frequency, and $\lambda_{e}=\sqrt{\frac{T_{e}}{4 \pi N e^{2}}}$, electron Debye length. Now the integral in Eq. (6) can be carried out by picking up the residue at the pole $k_{z}=i \gamma$ to yield

$D_{r}=1+\frac{1}{q(\omega)} \sqrt{\frac{q \kappa}{1+q \kappa}}=0 \quad\left(\kappa=k_{x}^{2} \lambda_{e}^{2}\right)$.

The quantity $\gamma$ is the attenuation constant, giving the $z$ dependence $\sim e^{-\gamma z}$. Clearly, as is seen from Eq. (7), $q(\omega)$ should be negative to have the ion acoustic surface wave supported. Also we need $1+q \kappa<0$ :

$q<0, q+\frac{1}{\kappa}<0$.

Squaring Eq. (7) gives $\kappa q^{2}+q-\kappa=0$ which is solved by

$q=\frac{1}{2 \kappa}\left(-1 \pm \sqrt{1+4 \kappa^{2}}\right)$.

Here we consider two limiting cases of $\kappa$. i) $\kappa<<1$ : In this case, we have $q \simeq \frac{1}{2 \kappa}\left[-1 \pm\left(1+2 \kappa^{2}\right)\right]$ in which the positive sign should be rejected in view of Eq. (8), while the (-) sign is in accord with Eq. (8). Neglecting the $\kappa^{2}$ term, we obtain

$\omega^{2}=\frac{k_{x}^{2} \lambda_{e}^{2} \omega_{p i}^{2}}{1+k_{x}^{2} \lambda_{e}^{2}} \quad\left(q \simeq-\frac{1}{\kappa}, \kappa<<1\right)$.

Equation (10) is the well-known dispersion relation. The surface ion acoustic wave dispersion relation is of the same form as the bulk ion acoustic wave.

ii) $\kappa \gg>1$ : In this case, we obtain $q \simeq \frac{1}{2 \kappa}(-1 \pm 2 \kappa)$. Only the (-) sign is acceptable and we have the solution

$\omega^{2}=\frac{\omega_{p i}^{2}}{2} \quad(q \simeq-1, \kappa>>1)$.

It should be noted that the surface ion acoustic wave does not asymptote to the line $\omega^{2}=\omega_{p i}^{2}$, but approaches the line $\omega^{2}=\frac{\omega_{p i}^{2}}{2}$.

We write for future reference

$\frac{\gamma}{k_{x}}=\sqrt{\frac{\kappa^{2}}{1+\kappa^{2}}}<<1$ when $\kappa<<1$,

$\frac{\gamma}{k_{x}}=\sqrt{\frac{2 \kappa-1}{2 \kappa+1}} \simeq 1$ when $\kappa>1$.

Next we calculate the imaginary part of Eq. (4):

$D_{i} \equiv-i \frac{k_{x}}{\pi} \int d k_{z} \frac{\varepsilon_{i}}{k^{2} \varepsilon_{r}^{2}}$.

Using the relation $k^{2} \varepsilon_{r}=q\left(k_{z}^{2}+\gamma^{2}\right)$ and Eq. (3), the above expression can be put into the form

$$
\begin{aligned}
& D_{i}=-i \frac{k_{x}}{\sqrt{\pi}} \frac{\omega}{q^{2}} \int_{-\infty}^{\infty} \frac{d k_{z}}{\left(k_{z}^{2}+\gamma^{2}\right)^{2} \sqrt{k_{z}^{2}+k_{x}^{2}}} \\
& \sum_{\alpha} \frac{1}{\lambda_{\alpha}^{2}} \sqrt{\frac{m_{\alpha}}{2 T_{\alpha}}} \exp \left[-\frac{m_{\alpha} \omega^{2}}{2 T_{\alpha}\left(k_{z}^{2}+k_{x}^{2}\right)}\right] .
\end{aligned}
$$

We first deal with the ion term, and consider integral

$I_{i}=\int_{-\infty}^{\infty} \frac{d k_{z} \exp \left[-\frac{\xi_{i}}{k_{z}^{2}+k_{x}^{2}}\right]}{\left(k_{z}^{2}+\gamma^{2}\right)^{2} \sqrt{k_{z}^{2}+k_{x}^{2}}}, \quad\left(\xi_{i}=\frac{m_{i} \omega^{2}}{2 T_{i}}\right)$.

We integrate Eq. (15) along the real $k_{z}$-axis without resorting to contour integration. It is useful to make a change of variable via

$y=\frac{k_{z}}{\sqrt{k_{z}^{2}+\gamma^{2}}}$, or $k_{z}=\frac{\gamma y}{\sqrt{1-y^{2}}}$. 
Then, integral $I_{i}$ becomes

$I_{i}=\frac{2}{\gamma^{3}} \int_{0}^{1} d y \frac{\left(1-y^{2}\right) \exp \left[-\frac{\xi_{i}\left(1-y^{2}\right)}{y^{2}\left(\gamma^{2}-k_{x}^{2}\right)+k_{x}^{2}}\right]}{\sqrt{y^{2}\left(\gamma^{2}-k_{x}^{2}\right)+k_{x}^{2}}}$.

Equation (17) is appropriate for asymptotic analysis for the limiting cases.

i) case of $\kappa<1$

In this case, we have $\gamma<k_{x}$. Neglecting $\gamma^{2}$ as compared to $k_{x}^{2}$, we obtain

$I_{i}=\frac{2}{k_{x} \gamma^{3}} e^{-\frac{\xi_{i}}{k_{x}^{2}}} I_{0}, \quad I_{0}=\int_{0}^{1} \sqrt{\left(1-y^{2}\right)} d y=\frac{\pi}{4}$.

Here we have $\frac{\xi_{i}}{k_{x}^{2}}=\frac{m_{i}}{2 T_{i}} \lambda_{e}^{2} \omega_{p i}^{2}=\frac{T_{e}}{2 T_{i}}$. Thus

$I_{i}=\frac{\pi}{2 k_{x} \gamma^{3}} e^{-\frac{T_{e}}{2 T_{i}}}$

The corresponding electron integral is calculated similarly by replacing $\xi_{i} \rightarrow \xi_{e}=\frac{m_{e} \omega^{2}}{2 T_{e}}$ in Eq. (17). Here $\frac{\xi_{e}}{k_{x}^{2}}=\frac{m_{e}}{2 m_{i}} \ll<1$ and the exponential term can be put to unity. We obtain

$I_{e}=\frac{\pi}{2 k_{x} \gamma^{3}}$

Then Eqs. (14), (18), (19), and the relations $q=-1 / \kappa, \omega=\omega_{p i} k_{x} \lambda_{e}, \gamma=\kappa k_{x}$ give

$D_{i}=-i \sqrt{\frac{\pi}{8}} \frac{1}{k_{x}^{4} \lambda_{e}^{4}}\left[\left(\frac{T_{e}}{T_{i}}\right)^{\frac{3}{2}} e^{-\frac{T_{e}}{2 T_{i}}}+\sqrt{\frac{m_{e}}{m_{i}}}\right]$.

The Landau damping rate is obtained from the formula

$\omega_{i}=-\frac{D_{i}(\omega)}{\partial D_{r}(\omega) / \partial \omega}$.

We calculate from Eq. (7):

$\frac{}{\omega}=\frac{\partial D_{r}}{\partial q} \frac{\partial q}{\partial \omega}$

$\frac{\partial D_{r}}{\partial q}=\frac{1}{q}-\frac{1}{2 \kappa} \simeq-\frac{1}{2 \kappa}, \quad \frac{\partial q}{\partial \omega} \simeq \frac{2}{\omega_{p i} k_{x}^{3} \lambda_{e}^{3}}$.

Using the above relations in the formula (21) yields the Landau damping rate for $\kappa<1$

$\omega_{i}=-\omega_{p i} k_{x} \lambda_{e} \sqrt{\frac{\pi}{8}}\left[\left(\frac{T_{e}}{T_{i}}\right)^{\frac{3}{2}} e^{-\frac{T_{e}}{2 T_{i}}}+\sqrt{\frac{m_{e}}{m_{i}}}\right]$.
In Eq. (22), the mass ratio term comes from the electron term in $\varepsilon_{i}$. It is interesting to see that surface ion acoustic wave Landau damping in Eq. (22) is equal to the corresponding Landau damping of the bulk ion acoustic wave [5]. In section 5, we explain about the analytical reason why the two damping rates are the same.

ii) case of $\kappa>>1$

In this case, we have $\gamma \simeq k_{x}$, so $I_{i}$ in Eq. (17) reduces to

$I_{i}=\frac{2}{k_{x} \gamma^{3}} e^{-\frac{\xi_{i}}{k_{x}^{2}}} J_{i}$

$J_{i}=\int_{0}^{1} d y\left(1-y^{2}\right) e^{\frac{\xi_{i} y^{2}}{k_{x}^{2}}}$.

Integral $J_{i}$ can be obtained by integration by parts and after some algebra:

$J_{i}=-\frac{1}{2 x^{2}} e^{x^{2}}+\frac{F(x)}{x}\left(1+\frac{1}{2 x^{2}}\right)$

where $F(x)$ is the error function:

$F(x)=\int_{0}^{x} e^{t^{2}} d t ; x=\frac{\sqrt{\xi_{i}}}{k_{x}}=\frac{1}{2} \sqrt{\frac{T_{e}}{T_{i}}} \frac{1}{k_{x} \lambda_{e}}$.

The quantity $x$ in Eq. (26), which is the ratio of the surface wave velocity to the ion thermal velocity, is assumed to be $>>1$ from the beginning when we wrote Eq. (3). The limiting conditions $x>>1$ and $k_{x} \lambda_{e}>>1$ restricts the validity of the present analysis $(\kappa>>1)$ to the regions

$\sqrt{\frac{T_{e}}{T_{i}}} \gg k_{x} \lambda_{e} \gg 1$.

Using Eq. (25) in Eq. (23) gives

$I_{i}=\frac{2}{k_{x} \gamma^{3}}\left[-\frac{1}{2 x^{2}}+\frac{e^{-x^{2}}}{x}\left(1+\frac{1}{2 x^{2}}\right) F(x)\right]$.

The error function $F(x)$ has asymptotic expansion for $x>1[6]$ :

$F(x)=\int_{0}^{x} e^{t^{2}} d t \simeq \frac{e^{x^{2}}}{2 x}\left(1+\frac{1}{2 x^{2}}+\frac{3}{4 x^{4}}+\cdots\right) \quad(x>>1)$.

The above two equations give, using $\gamma \simeq k_{x}$, $\omega=\omega_{p i} / \sqrt{2}$,

$I_{i} \simeq \frac{1}{k_{x} \gamma^{3}} \frac{1}{x^{4}}\left(1+\frac{1}{x^{2}}\right)=16\left(\frac{T_{i}}{T_{e}}\right)^{2} \lambda_{e}^{4}$.

Next we calculate the electron integral for this case of $\kappa>1$. We redefine the symbol $x$ :

$x=\frac{\sqrt{\xi_{e}}}{k_{x}}=\frac{1}{2} \sqrt{\frac{m_{e}}{m_{i}}} \frac{1}{k_{x} \lambda_{e}}<<1$ 
which is the ratio of the surface wave velocity to the electron thermal velocity. The electron integral corresponding to Eq. (15) is

$I_{e}=\frac{2}{k_{x} \gamma^{3}} e^{-x^{2}} J_{e}$

$J_{e}=\int_{0}^{1} d y\left(1-y^{2}\right) e^{\frac{\xi_{e} y^{2}}{k_{x}^{2}}}=-\frac{1}{2 x^{2}} e^{x^{2}}+\frac{F(x)}{x}\left(1+\frac{1}{2 x^{2}}\right)$

which is of the same form as Eq. (25), but $x$ is now $<<1$ and $F(x)$ is represented by different asymptotic series:

$F(x)=x e^{x^{2}}\left(1-\frac{2}{3} x^{2}+\frac{4}{15} x^{4}-\cdots\right) \quad(x<<1)$.

Using the above two equations in Eq. (30), we obtain simple result with neglect of higher order terms

$I_{e}=\frac{2}{k_{x} \gamma^{3}}$.

Using Eqs. (28) and (31) in Eq. (14) gives

$D_{i}=\frac{-8 i}{\sqrt{\pi}} \sqrt{\frac{T_{i}}{T_{e}}} k_{x} \lambda_{e}-\frac{i}{\sqrt{\pi}} \sqrt{\frac{m_{e}}{m_{i}}} \frac{1}{k_{x}^{3} \lambda_{e}^{3}}$.

To apply formula (21), we calculate

$\frac{\partial D_{r}}{\partial q} \simeq-1, \frac{\partial q}{\partial \omega} \simeq \frac{4 \sqrt{2}}{\omega_{p i}}$.

Using the above values, we obtain for Landau damping rate when $\kappa \gg>1$

$\omega_{i}=-\sqrt{\frac{2}{\pi}} \sqrt{\frac{T_{i}}{T_{e}}} \omega_{p i} k_{x} \lambda_{e}-\frac{1}{8} \sqrt{\frac{2}{\pi}} \sqrt{\frac{m_{e}}{m_{i}}} \frac{\omega_{p i}}{k_{x}^{3} \lambda_{e}^{3}} \equiv \omega_{i}^{i}+\omega_{i}^{e}$

where the superscripts denote ion and electron contribution from $\varepsilon_{i}$. The ratio of electron contribution to ion's is

$\frac{\omega_{i}^{i}}{\omega_{i}^{e}}=8 \sqrt{\frac{m_{i}}{m_{e}}} \sqrt{\frac{T_{i}}{T_{e}}} k_{x}^{4} \lambda_{e}^{4}>>1$.

So the electron term in $\varepsilon_{i}$ in Eq. (3) contributes negligibly to ion acoustic wave Landau damping when $k_{x} \lambda_{e} \gg 1$.

\section{SURFACE ELECTRON PLASMA WAVE}

For the high frequency wave $\left(\omega>>k \cdot v_{e}\right), \varepsilon_{L}=\varepsilon_{r}+i \varepsilon_{i}$ has the expression

$\varepsilon_{r}=1-\frac{\omega_{p e}^{2}}{\omega^{2}}-3 \frac{\omega_{p e}^{4}}{\omega^{4}} k^{2} \lambda_{e}^{2}$

$\varepsilon_{i}=\sqrt{\frac{\pi}{2}}\left(\frac{m_{e}}{T_{e}}\right)^{\frac{3}{2}} \frac{\omega \omega_{p e}^{2}}{k^{3}} \exp \left[-\frac{m_{e}}{2 T_{e}} \frac{\omega^{2}}{k^{2}}\right]$.

The real part of dispersion integral in Eq. (4) can be evaluated as follows

$$
\begin{aligned}
\int \frac{d k_{z}}{k^{2} \varepsilon_{r}} & =\int \frac{d k_{z}}{k^{2}\left(1-\frac{\omega_{p e}^{2}}{\omega^{2}}-3 \frac{\omega_{p e}^{4}}{\omega^{4}} k^{2} \lambda_{e}^{2}\right)} \\
& =\frac{-\omega^{4}}{3 \omega_{p e}^{4} \lambda_{e}^{2}} \int \frac{d k_{z}}{\left(k_{z}^{2}+k_{x}^{2}\right)\left(k_{z}^{2}+\gamma^{2}\right)} \\
\gamma & =\sqrt{k_{x}^{2}-\frac{\omega^{4}\left(1-\frac{\omega_{p e}^{2}}{\omega^{2}}\right)}{3 \omega_{p e}^{4} \lambda_{e}^{2}}} .
\end{aligned}
$$

Picking up the residues at the poles $k_{z}=i k_{x}$ and $k_{z}=i \gamma$, the integral has the value $\pi \cdot \frac{1 / k_{x}-1 / \gamma}{1-\omega_{p e}^{2} / \omega^{2}}$, and the real part of the dispersion relation reads

$$
D_{r}=1+\frac{1}{q(\omega)}\left(1-\frac{k_{x}}{\gamma}\right), q(\omega)=1-\frac{\omega_{p e}^{2}}{\omega^{2}} .
$$

The real frequency is obtained from $D_{r}=0$. We seek the solution with the condition $q<0$, which insures that the quantity in the square root of Eq. (37) is positive. Accordingly, we have $\gamma>k_{x}$. The relation $D_{r}=0$ gives after squaring

$$
-1+(2-x)^{2}-\frac{(1-x)(2-x)^{2}}{3 k_{x}^{2} \lambda_{e}^{2} x^{2}}=0 \quad\left(x=\frac{\omega_{p e}^{2}}{\omega^{2}}\right) .
$$

It is seen that $x=2$ is an approximate solution when $k_{x}^{2} \lambda_{e}^{2}<<1$. Note that $x=1$ or $q=0$ (in this case $\gamma=k_{x}$ ) is not a root of $D_{r}=0$. Let us look for a solution of the above equation in the vicinity of $x=2$. So put $x=2+\delta$ $(\delta<<1)$, and obtain $\delta$ in the region of $k_{x} \lambda_{e}<<1$. One obtains

$$
\omega=\frac{\omega_{p e}}{\sqrt{2}}\left(1+\frac{\sqrt{3}}{2} k_{x} \lambda_{e}\right) .
$$

This frequency is obtained in Ref. [2]. See also Ref. [7]. Using Eq. (39) in Eq. (37) gives

$\frac{k_{x}}{\gamma}=2 \sqrt{3} k_{x} \lambda_{e}<<1$.

Next we calculate the Landau damping corresponding to the mode (39). The imaginary part of the dispersion integral in Eq. (4) upon using Eqs. (35) and (36) takes the form

$$
\begin{gathered}
D_{i}=-i A I, \quad A=\frac{1}{9 \sqrt{2 \pi}} \sqrt{\frac{m_{e}}{T_{e}}} \frac{k_{x}}{\lambda_{e}^{6}} \frac{\omega^{9}}{\omega_{p e}^{8}} \\
I=\int_{-\infty}^{\infty} d k_{z} \frac{\exp \left[-\frac{\alpha}{k_{z}^{2}+k_{x}^{2}}\right]}{\left(k_{z}^{2}+k_{x}^{2}\right)^{\frac{5}{2}}\left(k_{z}^{2}+\gamma^{2}\right)^{2}} \quad\left(\alpha=\frac{m_{e} \omega^{2}}{2 T_{e}}\right) .
\end{gathered}
$$

It is useful to make a change of variable via 
$y=\frac{k_{z}}{\sqrt{k_{z}^{2}+k_{x}^{2}}}$ or $k_{z}=\frac{k_{x} y}{\sqrt{1-y^{2}}}$.

Then the integral $I$ in Eq. (42) becomes

$I=\frac{2}{k_{x}^{4}} e^{-x^{2}} \int_{0}^{1} d y\left(1-y^{2}\right)^{3} \frac{e^{x^{2} y^{2}}}{\left[k_{x}^{2} y^{2}+\gamma^{2}\left(1-y^{2}\right)\right]^{2}} \quad\left(x^{2}=\frac{\alpha}{k_{x}^{2}}\right)$. form

In the region where $k_{x}<<\gamma$, integral $I$ takes simple

$I=\frac{2}{k_{x}^{4} \gamma^{4}} e^{-x^{2}} J, \quad J=\int_{0}^{1} d y\left(1-y^{2}\right) e^{x^{2} y^{2}}$

$x=\frac{\sqrt{\alpha}}{k_{x}}=\frac{1}{2} \frac{1}{k_{x} \lambda_{e}}>>1$.

After integration by parts in Eq. (44), one obtains

$J=\frac{1}{x} F(x)\left(1+\frac{1}{2 x^{2}}\right)-\frac{1}{2 x^{2}} e^{x^{2}} \quad(E q \cdot(25))$.

Using the asymptotic series for the error function $F(x)(x>>1)$, we obtain

$J=\frac{e^{x^{2}}}{2 x^{4}}\left(1+\frac{1}{x^{2}}\right)$.

Using Eqs. (45) and (46) in Eq. (44) yields

$I=\frac{1}{k_{x}^{4} \gamma^{4} x^{4}}\left(1+\frac{1}{x^{2}}\right)=9 \cdot 2^{8} \cdot \lambda_{e}^{8}\left(1+4 k_{x}^{2} \lambda_{e}^{2}\right)$

where the last term will be neglected. Then Eq. (41) gives

$D_{i}=-i \frac{8}{\sqrt{\pi}} k_{x} \lambda_{e}$.

Landau damping rate will be obtained by the formula (21). Equation (38) gives

$\frac{\partial D_{r}}{\partial \omega}=-\frac{1}{q^{2}} \frac{\partial q}{\partial \omega}\left(1-\frac{k_{x}}{\gamma}\right)+\frac{k_{x}}{q} \frac{1}{\gamma^{2}} \frac{\partial \gamma}{\partial \omega}$.

We have $\frac{\partial q}{\partial \omega} \simeq \frac{4 \sqrt{2}}{\omega_{p e}}, q \simeq-1, \gamma>>k_{x}$. The first term of Eq. (48) is $\simeq-\frac{4 \sqrt{2}}{\omega_{p e}}$, and the second term is $\simeq \frac{12 \sqrt{2}}{\omega_{p e}} k_{x}^{2} \lambda_{e}^{2}$ which will be neglected. So we finally obtain for the Landau damping rate

$\omega_{i}=-\sqrt{\frac{2}{\pi}} k_{x} \lambda_{e} \omega_{p e}$.

\section{TRANSVERSE MAGNETIC MODE SURFACE WAVE}

The transverse magnetic (TM) mode surface wave is known as surface polariton in solid state physics and a wave of partly electromagnetic and partly electrostatic nature whose kinetic dispersion relation is [1]

$$
D \equiv 1+\frac{1}{\pi} \frac{1}{\sqrt{k_{x}^{2}-\frac{\omega^{2}}{c^{2}}}} \int_{-\infty}^{\infty} \frac{d k_{z}}{k^{2}}\left(\frac{k_{x}^{2}}{\varepsilon_{L}}+\frac{k_{z}^{2}}{\varepsilon_{T}-c^{2} k^{2} / \omega^{2}}\right)=0
$$

where the longitudinal dielectric function $\varepsilon_{L}$ is expressed by Eqs. (35) and (36) which are rewritten:

$$
\varepsilon_{L}=1-\frac{\omega_{p e}^{2}}{\omega^{2}}-3 \frac{\omega_{p e}^{4}}{\omega^{4}} k^{2} \lambda_{e}^{2}+i \sqrt{\frac{\pi}{2}} \frac{\omega_{p e}^{2}}{\omega^{2}} \frac{\omega^{3}}{k^{3}}\left(\frac{m_{e}}{T_{e}}\right)^{\frac{3}{2}} \exp \left(-\frac{m_{e} \omega^{2}}{2 T_{e} k^{2}}\right)
$$

where the wave velocity is such that $\omega>>k \cdot v_{e}$. The transverse dielectric function $\varepsilon_{T}$ for such a fast wave is represented by [1]

$$
\varepsilon_{T}=1-\frac{\omega_{p e}^{2}}{\omega^{2}}-\frac{\omega_{p e}^{4}}{\omega^{4}} k^{2} \lambda_{e}^{2}+i \sqrt{\frac{\pi}{2}} \frac{\omega_{p e}^{2}}{\omega^{2}} \frac{\omega}{k}\left(\frac{m_{e}}{T_{e}}\right)^{\frac{1}{2}} \exp \left(-\frac{m_{e} \omega^{2}}{2 T_{e} k^{2}}\right) .
$$

The real part of the dispersion function $D$ is determined by the real parts of $\varepsilon_{L}$ and $\varepsilon_{T}$ :

$$
D_{r}=1+\frac{1}{\pi} \frac{1}{\sqrt{k_{x}^{2}-\frac{\omega^{2}}{c^{2}}}} \int_{-\infty}^{\infty} \frac{d k_{z}}{k^{2}}\left(\frac{k_{x}^{2}}{\varepsilon_{L}^{r}}+\frac{k_{z}^{2}}{\varepsilon_{T}^{r}-c^{2} k^{2} / \omega^{2}}\right)
$$

where the superscript ' $r$ ' denotes the real part. Assuming $\varepsilon_{L}^{r} \gg \varepsilon_{L}^{i}, \quad \varepsilon_{T}^{r} \gg \varepsilon_{T}^{i}$ (the superscript ' $i$ ' denotes the imaginary part), the imaginary part of the dispersion function is written

$$
D_{i}=\frac{-i}{\pi} \frac{1}{\sqrt{k_{x}^{2}-\frac{\omega^{2}}{c^{2}}}} \int_{-\infty}^{\infty} \frac{d k_{z}}{k^{2}}\left(\frac{k_{x}^{2} \varepsilon_{L}^{i}}{\left(\varepsilon_{L}^{r}\right)^{2}}+\frac{k_{z}^{2} \varepsilon_{T}^{i}}{\left(\varepsilon_{T}^{r}-c^{2} k^{2} / \omega^{2}\right)^{2}}\right) .
$$

The real frequency of the wave is determined by $D_{r}=0$. To carry out the integral in Eq. (53), we write

$$
\begin{aligned}
& \varepsilon_{L}^{r}=q \frac{\gamma_{L}^{2}+k_{z}^{2}}{\gamma_{L}^{2}-k_{x}^{2}}, \quad \varepsilon_{T}^{r}-\frac{c^{2} k^{2}}{\omega^{2}}=q \frac{\gamma_{T}^{2}+k_{z}^{2}}{\gamma_{T}^{2}-k_{x}^{2}} \\
& q=1-\frac{\omega_{p e}^{2}}{\omega^{2}}, \quad \gamma_{L}=\sqrt{k_{x}^{2}-\frac{q \omega^{4}}{3 \omega_{p e}^{4} \lambda_{e}^{2}}}, \quad \gamma_{T}=\sqrt{k_{x}^{2}-\frac{q \omega^{4}}{\omega_{p e}^{4} \lambda_{e}^{2}+c^{2} \omega^{2}}} .
\end{aligned}
$$

Then Eq. (53) is contour-integrated to yield

$$
D_{r}=1+\frac{1}{\sqrt{k_{x}^{2}-\frac{\omega^{2}}{c^{2}}}} \frac{1}{q \gamma_{L}}\left(\gamma_{L} \gamma_{T}-k_{x}^{2}\right) .
$$

Using Eq. (55) in Eq. (54) gives

$$
\begin{aligned}
D_{i} & =\frac{-i}{\pi} \frac{1}{\sqrt{k_{x}^{2}-\frac{\omega^{2}}{c^{2}}}}\left(T_{L}+T_{T}\right) \\
T_{L} & =\sqrt{\frac{\pi}{2}} \omega \omega_{p e}^{2}\left(\frac{m_{e}}{T_{e}}\right)^{\frac{3}{2}} \frac{\left(\gamma_{L}^{2}-k_{x}^{2}\right)^{2}}{q^{2}} k_{x}^{2} I_{L}
\end{aligned}
$$


$T_{T}=\sqrt{\frac{\pi}{2}} \frac{\omega_{p e}^{2}}{\omega}\left(\frac{m_{e}}{T_{e}}\right)^{\frac{1}{2}} \frac{\left(\gamma_{T}^{2}-k_{x}^{2}\right)^{2}}{q^{2}} I_{T}$

$I_{L}=\int_{-\infty}^{\infty} d k_{z} \frac{\exp \left(-\frac{m_{e} \omega^{2}}{2 T_{e}\left(k_{z}^{2}+k_{x}^{2}\right)}\right)}{\left(k_{z}^{2}+k_{x}^{2}\right)^{\frac{5}{2}}\left(k_{z}^{2}+\gamma_{L}^{2}\right)^{2}}$

$I_{T}=\int_{-\infty}^{\infty} d k_{z} \frac{k_{z}^{2} \exp \left(-\frac{m_{e} \omega^{2}}{2 T_{e}\left(k_{z}^{2}+k_{x}^{2}\right)}\right)}{\left(k_{z}^{2}+k_{x}^{2}\right)^{\frac{3}{2}}\left(k_{z}^{2}+\gamma_{T}^{2}\right)^{2}}$.

The eigen frequency of the electromagnetic surface wave can be found by solving the equation $D_{r}=0$. However the algebraic equation is unwieldy, and we consider the limiting case of $k_{x} \lambda_{e}<<1$. In this case $\gamma_{L} \gamma_{T} \gg k_{x}^{2}$, and the dispersion relation, Eq. (57), reduces to

$D_{r}=1+\frac{1}{\sqrt{k_{x}^{2}-\frac{\omega^{2}}{c^{2}}}} \frac{\gamma_{T}}{q}=0$.

We see that $q$ should be negative for the above equation to be satisfied. Also the condition insures the quantities in the square root of $\gamma_{T, L}$ be positive. When $\lambda_{e} \rightarrow 0$ (cold plasma limit), Eq. (63) takes the form

$\left(1-\frac{\omega_{p e}^{2}}{\omega^{2}}\right) \sqrt{k_{x}^{2}-\frac{\omega^{2}}{c^{2}}}+\sqrt{k_{x}^{2}+\frac{\omega_{p e}^{2}-\omega^{2}}{c^{2}}}=0$.

It is fortunate that Eq. (64) allows for exact solution by quadrature after squaring [8]:

$\omega^{2}=c^{2} k_{x}^{2}+\frac{\omega_{p e}^{2}}{2}-\frac{1}{2} \sqrt{\omega_{p e}^{4}+4 c^{4} k_{x}^{4}}$.

It is interesting to note that Eq. (65) is identical in the structure with the surface ion acoustic wave dispersion relation

$\omega^{2}=\omega_{p i}^{2} \lambda_{e}^{2} k_{x}^{2}+\frac{\omega_{p i}^{2}}{2}-\frac{1}{2} \sqrt{\omega_{p i}^{4}+4 \omega_{p i}^{4} \lambda_{e}^{4} k_{x}^{4}}$.

which is the solution of Eq. (9). The plot of Eq. (65) is available in Alexandrov et al. (figure 9.2) [1].

Case (i): $\omega_{p e}^{2} \gg 2 c^{2} k_{x}^{2}$. The dispersion curve, frequency $\omega$ vs $c k_{x}$, shows linear increase, starting from $c k_{x}=0$. We have from Eq. (65)

$$
\begin{aligned}
& \omega^{2} \simeq c^{2} k_{x}^{2}\left(1-2 \frac{c^{2} k_{x}^{2}}{\omega_{p e}^{2}}\right), \quad q \simeq-\frac{\omega_{p e}^{2}}{c^{2} k_{x}^{2}} \\
& \frac{\gamma_{L}}{k_{x}} \simeq \frac{1}{\sqrt{3}} \frac{c}{v_{e}}>1, \frac{\gamma_{T}}{k_{x}} \simeq\left[\frac{v_{e}^{2}}{c^{2}}+\frac{c^{2} k_{x}^{2}}{\omega_{p e}^{2}}\right]^{-\frac{1}{2}} \gg 1
\end{aligned}
$$

where $v_{e}^{2}=\frac{T_{e}}{m_{e}}$, electron thermal velocity squared.

Case (ii): $\omega_{p e}^{2} \ll<2 c^{2} k_{x}^{2}$. This case corresponds to the nearly flat portion of the dispersion curve which asymptotes to the line $\omega=\omega_{p e} / \sqrt{2}$.

$$
\begin{aligned}
& \omega^{2} \simeq \frac{\omega_{p e}^{2}}{2}\left(1-\frac{\omega_{p e}^{2}}{4 c^{2} k_{x}^{2}}\right), q \simeq-1 \\
& \frac{\gamma_{L}}{k_{x}} \simeq \frac{1}{\sqrt{12} k_{x} \lambda_{e}}>>1, \frac{\gamma_{T}}{k_{x}} \simeq \sqrt{1+\frac{\omega_{p e}^{2}}{2 c^{2} k_{x}^{2}}} \simeq 1 .
\end{aligned}
$$

Guided by the cold plasma solution, we shall consider the integrals in Eqs. (61) and (62) corresponding to the limiting cases (i) and (ii).

Case (i). $I_{L}$ in Eq. (61) is identical with Eq. (42) with $\gamma_{L}=\gamma$ in Eq. (37). So we can use the result in section 3,

$I_{L}=\frac{1}{k_{x}^{4} \gamma_{L}^{4} x^{4}}, \quad x^{2}=\frac{m_{e} \omega^{2}}{2 T_{e} k_{x}^{2}}=\frac{c^{2} m_{e}}{2 T_{e}}$.

Using Eq. (67) in the above gives

$I_{L}=36 \frac{T_{e}^{4}}{m_{e}^{4} c^{8} k_{x}^{8}}$

which in turn gives

$T_{L}=\sqrt{8 \pi} k_{x} \lambda_{e}^{2} k_{x}^{2} \frac{c}{\sqrt{T_{e} / m_{e}}}$.

Next, let us calculate $I_{T}$. Since we have $\gamma_{T}>k_{x}$, we can use the same transform as that used in Eq. (42), giving

$I_{T}=\frac{2}{\gamma_{T}^{4}} e^{-x^{2}} \int_{0}^{1} \frac{y^{2}}{1-y^{2}} e^{x^{2} y^{2}} d y$.

The integral above can be asymptotically evaluated to obtain the value $\simeq x^{-2} e^{x^{2}}$ for the leading term, giving

$I_{T}=\frac{2}{x^{2} \gamma_{T}^{4}}$.

Using Eqs. (67) and (73) in Eq. (60) yields

$$
T_{T}=\sqrt{8 \pi} k_{x} \lambda_{e}^{2} k_{x}^{2} \frac{c}{\sqrt{T_{e} / m_{e}}}
$$

which is the same as $T_{L}$. Using Eqs. (71) and (74) in Eq. (58) gives

$$
D_{i}=\frac{4}{\sqrt{\pi}} \lambda_{e} k_{x}
$$

which is valid in the region $c k_{x} \ll \omega_{p e} / \sqrt{2}$.

Case (ii). To evaluate $I_{L}$ in Eq. (61) in this case, we note that $\gamma_{L} \gg k_{x}$ (Eq. (68)). So using the result in section 3, we have 
$I_{L}=\frac{1}{k_{x}^{4} \gamma_{L}^{4} x^{4}}, \quad x^{2}=\frac{1}{4 k_{x}^{2} \lambda_{e}^{2}}$

Using Eq. (68) in the above gives

$I_{L}=16 \cdot 12^{2} \cdot \lambda_{e}^{8}$

which is used in Eq. (59) to obtain

$T_{L}=8 \sqrt{\pi} \lambda_{e} k_{x}^{2}$.

Next, to evaluate $I_{T}$ in Eq. (62), we transform the integral as in Eq. (42). Since we have $\gamma_{T} \simeq k_{x}, I_{T}$ takes the value

$I_{T}=\frac{2}{\gamma_{T}^{4}} e^{-x^{2}} \int_{0}^{1} y^{2}\left(1-y^{2}\right) e^{x^{2} y^{2}} d y \simeq \frac{2}{\gamma_{T}^{4}} e^{-x^{2}} \cdot \frac{e^{x^{2}}}{2 x^{4}}=\frac{1}{\gamma_{T}^{4} x^{4}}$

where $x^{2}$ is given by Eq. (76). Using Eqs. (68) and (79) in Eq. (60) yields

$T_{T}=4 \sqrt{\pi} \frac{v_{e}^{4}}{c^{4}} \frac{1}{\lambda_{e}}$.

Using Eqs. (78) and (80) in Eq. (58) gives

$D_{i}=\frac{8}{\sqrt{\pi}} k_{x} \lambda_{e}\left(1+\frac{1}{2} \frac{v_{e}^{4}}{c^{4}} \frac{1}{k_{x}^{2} \lambda_{e}^{2}}\right)$.

The second term in the parenthesis is not necessarily small because it is a product of a small quantity $\left(v_{e}^{4} / c^{4}\right)$ and a large quantity $\left(1 / k_{x}^{2} \lambda_{e}^{2}\right)$.

Next, in order to calculate $\partial D_{r} / \partial \omega$ we use Eqs. (63) and (56) to obtain

$\frac{\partial D_{r}}{\partial \omega}=\frac{1}{q} \frac{1}{\sqrt{k_{x}^{2}-\frac{\omega^{2}}{c^{2}}}}\left[\frac{\omega}{c^{2}} \frac{\gamma_{T}}{\left(k_{x}^{2}-\omega^{2} / c^{2}\right)}-\frac{2 \gamma_{T} \omega_{p e}^{2}}{q \omega^{3}}+\frac{\partial \gamma_{T}}{\partial \omega}\right]$

$\frac{\partial \gamma_{T}}{\partial \omega}=-\frac{\omega}{\gamma_{T}} \frac{\omega_{p e}^{4} \lambda_{e}^{2}\left(2 \omega^{2}-\omega_{p e}^{2}\right)+c^{2} \omega^{4}}{\left(\omega_{p e}^{4} \lambda_{e}^{2}+c^{2} \omega^{2}\right)^{2}}$.

Equations (82) and (83) will be evaluated according to the conditions of cases (i) and (ii) expressed in Eqs. (67) and (68).

Case (i): The last two terms in the large bracket in Eq. (82) add up to yield a simple result $3 \gamma_{T} / \omega$ which in turn is negligible as compared to the first term. Thus, we obtain

$\frac{\partial D_{r}}{\partial \omega}=-\frac{1}{2 \sqrt{2}} \frac{\omega_{p e}}{c^{2} k_{x}^{2}}\left(\frac{v_{e}^{2}}{c^{2}}+\frac{c^{2} k_{x}^{2}}{\omega_{p e}^{2}}\right)^{-\frac{1}{2}}$. (84):

The Landau damping rate is obtained with Eqs. (75) and

$$
\omega_{i}=-8 \sqrt{\frac{2}{\pi}} \omega_{p e}\left(k_{x} \lambda_{e}\right)^{4} \frac{c^{3}}{v_{e}^{3}} \sqrt{1+\frac{v_{e}^{4}}{c^{4}} \frac{1}{k_{x}^{2} \lambda_{e}^{2}}} .
$$

This result is valid around the linear portion of the dispersion curve.

Case (ii): The first term and the third term in the large bracket in Eq. (82) cancel. Due to the sole contribution from the second term, we have

$\frac{\partial D_{r}}{\partial \omega}=-\frac{4 \sqrt{2}}{\omega_{p e}}$.

The Landau damping rate in this case is obtained with Eqs. (81) and (86):

$\omega_{i}=-\sqrt{\frac{2}{\pi}} \omega_{p e} k_{x} \lambda_{e}\left(1+\frac{1}{2} \frac{v_{e}^{4}}{c^{4}} \frac{1}{k_{x}^{2} \lambda_{e}^{2}}\right)$.

This result is valid on the flat portion of the dispersion curve.

\section{DISCUSSION} works.

We compare the results obtained in this work with earlier

Concerning ion acoustic wave, Landau damping rate in Eq. (22) for the case of $k_{x} \lambda_{e}<<1$ is identical with Equation (11.29a) of Sitenko [4]. Sitenko's Eq. (11.29b) (for the case $k_{x} \lambda_{e}>>1$ ) [4] is identical with the first term of Eq. (34) with a numerical difference of factor $\sqrt{3}$. The second term of Eq. (34) agrees with Alexandrov et al.'s equation (9.1.40) [1] with a small discrepancy of numerical factor. It appears that the electron term in $\varepsilon_{i}$ in Eq. (3) can be safely neglected in the consideration of ion acoustic wave damping. The electron plasma wave damping in Eq. (49) agrees with the results of Alexandrov et al. (Eq. 9.1.34) [1] and Sitenko (Eq. 11.27) [4].

We can see how the two Landau damping rates of bulk and surface ion waves for the case of $k_{x} \lambda_{e}<<1$ are the same from the following equation:

$\frac{D_{i}}{\partial D_{r} / \partial \omega}=\int \frac{d k_{z}}{k^{2}} \frac{\varepsilon_{i}}{\varepsilon_{r}^{2}} \div \int \frac{d k_{z}}{k^{2}} \frac{\frac{\partial \varepsilon_{r}}{\partial \omega}}{\varepsilon_{r}^{2}}=\frac{\varepsilon_{i}\left(k_{x}, \omega\right)}{\partial \varepsilon_{r}\left(k_{x}, \omega\right) / \partial \omega}$

If we make change of variable by the transform in Eq. (16), we have $k^{2} \simeq k_{x}^{2}$ for $k_{x} \gg \gamma$, and thus $\varepsilon_{i}(k, \omega)$ inside the integral can be taken out of the integral with the value $\varepsilon_{i}\left(k_{x}, \omega\right) . \frac{\partial \varepsilon_{r}}{\partial \omega}$ is also independent of $k_{z}$. Hence the above equation holds. The surface ion acoustic wave in the region $k_{x} \lambda_{e}<<1$ obeys the same dispersion relation as the bulk wave (Eq. (10)) and has attenuation constant much smaller than $k_{x}$. The surface ion wave is almost indistinguishable from the bulk ion acoustic wave and Landau damps with the same rate as the bulk wave.

Surface wave Landau damping is interesting not only on its own right but also for study of nonlinear Landau damping of surface wave which is the basic mechanism of a certain parametric instability. Stenflo [9] addresses nonlinear 
problem of surface waves in semi-infinite plasma, including three wave surface wave interactions.

\section{ACKNOWLEDGMENTS}

This work is supported by the Basic Science Research Program through the National Research Foundation of Korea (NRF) funded by the Ministry of Education, Science and Technology (MEST) (2011-0005112).

\section{CONFLICT OF INTEREST}

None declared.

\section{REFERENCES}

[1] Alexandrov AF, Bogdankevich LS, Rukhadze AA. Principles of plasma electrodynamics. Berlin: Springer 1984.
[2] Barr HC, Boyd TJM. Surface waves in hot plasmas. J Phys A 1972; 5: 1108-18.

[3] Lee HJ, Lim YK. Kinetic theory of surface waves in a plasma slab. J Korean Phys Soc 2007; 50: 1056-61.

[4] Sitenko AG. Fluctuations and Non-linear Wave Interactions in Plasmas. New York: Pergamon 1982; p. 231.

[5] Krall NA, Trivelpiece AW. Principles of Plasma Physics. New York: McGraw-Hill 1973.

[6] Bekefi G, Radiation processes in plasmas. New York: Wiley 1966.

[7] Kaw PK, McBride JB. Surface waves on a plasma. Phys Fluids 1970; 13: 1784-93.

[8] Lee HJ. Korteweg-de Vries equation of transverse magnetic mode of plasma surface wave. Plasma Phys Control Fusion 2004; 46: 447-53.

[9] Stenflo L. Theory of nonlinear plasma surface waves. Phys Scr 1996; T63: 59-62. 